\title{
Effects of ovariectomy on periodontal tissues following tooth replantation
}

\author{
Heloisa Fonseca Marão ${ }^{\mathrm{a}, *}$, Jeremy J. Mao ${ }^{\mathrm{b}}$, Cláudio Aparecido Casatti $^{\mathrm{c}, \mathrm{d}}$, \\ Paulo G. Coelho ${ }^{\mathrm{e}, \mathrm{f}, \mathrm{g}}$, Edilson Ervolino ${ }^{\mathrm{c}}$, Jian Zhou ${ }^{\mathrm{b}}$, Vanessa Ferreira da Silva ${ }^{\mathrm{a}}$, \\ Sônia Regina Panzarini ${ }^{\mathrm{a}}$
}

\author{
a Department of Surgery and Integrated Clinics, Aracatuba Dental School, Univ Estadual Paulista-UNESP, Rua José Bonifácio, 1193-Vila Mendonça, Aracatuba, \\ SP 16015-050, Brazil

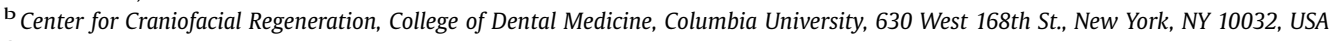 \\ ${ }^{c}$ Department of Basic Science, Aracatuba Dental School, Univ Estadual Paulista-UNESP, Rodovia Marechal Rondon, KM 527/528, Campus Universitário, \\ Aracatuba, SP 16018-805, Brazil \\ d Biosciences Institute of Botucatu, UNESP Univ Estadual Paulista, Botucatu, SP, Brazil \\ e Department of Biomaterials and Biomimetics, New York University College of Dentistry, 433 1st Ave., New York, NY 10010, USA \\ ${ }^{\mathrm{f}}$ Department of Periodontology and Implant Dentistry, New York University College of Dentistry, 345 E 24th St, New York, NY 10010, USA \\ ${ }^{\mathrm{g}}$ Division of Engineering, New York University Abu Dhabi, Abu Dhabi, United Arab Emirates
}

\section{A R T I C L E I N F O}

Article history:

Received 21 May 2015

Received in revised form 21 December 2015

Accepted 16 January 2016

\section{Keywords:}

Tooth replantation

Ovariectomy

Immunohistochemistry

Osteoclast

Micro-ct

\begin{abstract}
A B S T R A C T
Objectives: The aim of the study was to analyze the effects of ovariectomy on periodontal tissues following immediate tooth replantation by histomorphometric, immunohistochemistry, and $\mu \mathrm{CT}$ analysis.

Materials and methods: Eighty wistar rats (Rattus norvegicus albinos) with normal estrous cycles were randomly divided into two groups: ovariectomized (OVX) and Sham. Two months after surgery, the rats' upper right incisor was extracted followed by immediate reimplantation. The animals were sacrificed after 28, 45, and 60 days healing time. Histomorphometric and immunohistochemical analysis were performed by evaluation of PCNA and TRAP straining.

Results: The periodontal ligament was reinserted into the bone and cementum in the both groups. The immunohistochemical analysis revealed PCNA positive cells on the periodontal ligament in both groups at 28 days. Root resorption was noted at 45 days with immunoreactive cells for TRAP present in bone and tooth surface however no statistical differences between the groups were noticed. Histomorphometric analysis showed significant difference between groups in the periodontal ligament and root resorption parameters for the sub-items: intensity of chronic inflammatory infiltrate at 60 days $(p<0.01)$, the organization of the periodontal ligament at 28 days $(p<0.05)$, depth of root resorption at 45 days $(p<0.05)$ and at 60 days $(p<0.001)$. The $\mu \mathrm{CT}$ analysis showed multiple areas of bone resorption in association with OVX at 28 and 60 days with no significant differences between times in vivo. Conclusion: The ovariectomy did not have significant influence in periodontal tissue parameters following tooth reimplantation.
\end{abstract}

(c) 2016 Elsevier Ltd. All rights reserved.

\section{Introduction}

Avulsion of permanent teeth, while not very frequent, is one of the main sequelae of dental trauma due to its esthetic and functional impact on the patient (Panzarini et al., 2013). Identifying

\footnotetext{
* Corresponding author at: Rua Jose Bonifacio Numero 1193, Vila Mendonca, Aracatuba, Sao Paulo CEP: 16015-050, Brazil.

E-mail addresses: heloisafonsecamarao@yahoo.com.br, hellobba@hotmail.com (H.F. Marão).
}

the periodontal healing pattern remains a challenge, especially after reimplantation procedures.

The success of tooth reimplantation depends on the maintenance of vitality of the periodontal ligament, which allows the avulsed tooth to recover their functions (Andreasen, Borun, Jacobsen, \& Adreasen, 1995). Resorption is the main cause of failure of reimplantation and the prognosis is related to the type of resorption that may lead to complete destruction of the root (Panzarini et al., 2013).

Root resorption is basically classified as follows: surface resorption which may occur even in the absence of a significant 
inflammatory process; inflammatory resorption, which occurs in the presence of inflammatory connective tissue; and replacement resorption, in which the periodontal ligament is resorbed or removed and replaced by bone tissue (Andreasen et al., 1995).

Degeneration of the periodontal ligament depends on several factors, such as trauma, management of the root, extra-alveolar period and storage medium and pulp necrosis (Panzarini et al., 2013; Marão et al., 2012). In addition, in certain systemic diseases, such deficiency of estrogen could interfere in the periodontal tissue phenotypes.

Estrogen is known to play an important role in regulating bone homeostasis and preventing postmenopausal bone loss (Girasole, Passeri, Pedrazzoni, Giuliani, \& Passeri, 1995). The lack of estrogen causes an imbalance in bone remodeling, causing the rate of bone resorption to exceed the bone formation (Riggs, Khosla, \& Melton, 2002; Luvizuto, Dias, Queiroz, Okamoto, \& Okamoto, 2010).

The regulation of bone homeostasis occurs by the balance of protein expression (Jabbar et al., 2011) and pro-inflammatory cytokines (Zhou, Fu, Li, \& Qi, 2009) such as interleukin (IL)-1, IL-6, tumor necrosis factor-alpha (TNF- $\alpha$ ), and protein receptor activator of nuclear factor-k B ligand (RANKL), which have been identified suggesting the impact on bone resorption in periodontal tissues (Pfeilschifter, Koditz, Pfohl, \& Schatz, 2002).

Estrogen may play an important role in hold antiresorptive effects on alveolar bone, at least in part by increasing the expression level of osteoprotegerin (OPG) versus that of RANKL via estrogen receptor $\beta$ in human periodontal ligament cells (Liang, Yu, Wang, \& Ding, 2008). The interaction between RANKL and the receptor activator of nuclear factor-k B (RANK) has been shown to be required for osteoclast differentiation (Theoleyre et al., 2004). The imbalance between RANK/RANKL/OPG leads to activity of osteoclasts assessed by an increase in tartrate-resistant acid-phosphatase (TRAP) staining (Said et al., 2012). Because multinuclear TRAP-positive cells resorb bone, TRAP is a specific osteoclast differentiation marker (Kwak et al., 2010). On the other hand, $17 \beta$-estradiol could promote the proliferation and viability of osteoblastic MC3T3-E1 cells, associated with proliferation cell nuclear antigen (PCNA) mRNA expression, and also could stimulate osteoblastic differentiation and bone formation as assessed by alkaline phosphatase (Song, Zhang, \& Zhou, 2011).

Understanding the circumstances that lead to the regeneration of oral tissues has been a major challenge for dental research. It is known that a great variety of signals are released when an injury occurs, inducing neighboring cell populations to respond with proliferation, migration, or differentiation (Andreasen and Andreasen, 2007). Despite abundant work on the effects of estrogen deficiency in appendicular bone, the method by which multiple tissue phenotypes in the periodontium respond to ovariectomy and tooth reimplantation is poorly understood. In this study we established an animal model to analyze how estrogen deficiency affects multiple periodontal tissue phenotypes following tooth reimplantation.

\section{Materials and methods}

\subsection{Animal care and ethics}

The research protocol was approved by the Animal Care and Use Committee of the Aracatuba Dental School, UNESP-Universidade Estadual Paulista, Aracatuba, Sao Paulo, Brazil (protocol \# 2008003266).

Eighty adult female Wistar rats (Rattus norvegicus, albinus) weighing between 150 and $250 \mathrm{~g}$ were selected for the study. The animals were housed in plastic cages under climate-controlled conditions ( $12 \mathrm{~h}$ light $/ 12 \mathrm{~h}$ dark; thermostatically regulated room temperature) and were fed a standard solid chow (Racao Ativada
Produtor; Anderson \& Clayton S.A. Industria e Comercio, Sao Paulo, SP, Brazil) and water ad libitum, except for the 12 preoperative hours. The rats were selected for the experiment after the regular estrous cycle confirmation.

\subsection{Study design and surgical procedure}

The animals were randomly divided into two groups $(N=10$ per group) and submitted to either fictitious surgery (Sham) or ovariectomy (OVX) surgery under general anesthesia with xylazine hydrochloride (Dopaser, Laboratorio Calier do Brasil Ltda., Osasco, SP, Brazil; $0.03 \mathrm{~mL}$ per $100 \mathrm{~g}$ body weight) and ketamine hydrochloride (Dopalen; AgriBrands do Brasil Ltda.; $0.07 \mathrm{~mL}$ per $100 \mathrm{~g}$ body weight). Fifteen days after ovariectomy, all animals were subjected to the estrous cycle during seven consecutive days to confirm estrogen depletion.

\subsection{Tooth extraction}

Two months after the surgical procedure, the animals were anesthetized and the anterior maxilla asepsis was performed with $1 \%$ iodine polyvinylpyrrolidone (Riodeine; Industria Farmaceutica Rioquimica Ltda., Sao Jose do Rio Preto, SP, Brazil), followed by extraction of the right maxillary incisor and immediate tooth reimplantation.

\subsection{Tissue processing}

The rats were sacrificed by anesthetic overdose after 28, 45 and 60 days and the perfusion (Cole-Parmer Instrument Company, Vernon Hills, IL, USA) was performed with the infusion of $4 \%$ formaldehyde (Acros Organics, NJ, USA) to remove the right maxilla. The anatomic pieces obtained were fixed in $4 \%$ formaldehyde, demineralized in a 10\% EDTA solution, pH 7\% (SigmaAldrich) and embedded in paraffin. Semi-serial longitudinal $5 \mu \mathrm{m}$ thick sections were obtained and divided interchangeably for histological (hematoxylin and eosin staining) and immunohistochemical analysis.

\section{5. $\mu C T$ and $3 D$ reconstruction}

For the $\mu \mathrm{CT}$ analysis, the early postoperative time point of 28 days $(n=5)$ and the final postoperative time point of 60 days $(n=5)$ were concerned. The harvested maxillae were removed from the formaldehyde, washed and stored at 70\% ethanol and separated from the anatomic pieces. A vertical cut was performed on the distal third molar, exposing both the area of the jaw containing the reimplanted tooth and the native tooth (control).

These samples were examined using $\mu \mathrm{CT}$ ( $\mu \mathrm{CT} 40$, Scanco Medical, Basserdorf, Germany) at $50 \mathrm{kV} / 160 \mu \mathrm{A}$, with an integration time of $1 \times 380 \mathrm{~ms}$ and a high resolution of $18 \mu \mathrm{m}$. Volumetric data was converted to DICOM format and imported in Amira program (Visage Imaging $\mathrm{GmbH}$, Berlin, Germany), which allowed the quantification of the root resorption extension, the periodontal ligament space, the bone resorption and the depth of root resorption. All the $\mu \mathrm{CT}$ measurements were performed by blind examiners and by one investigator.

The data were submitted to statistic analysis using analysis of variance (ANOVA) followed by a post hoc Mann-Whitney test. The significance level was set up at $5 \%$.

\subsection{Histomorphometric analysis}

The analysis of the outcomes was performed by one of the authors in a blind fashion, according to 16 histomorphometric parameters listed by Holland et al. (1999), Panzarini et al. (2007), 
Marão et al. (2012). The histomorphometric parameters have been used to immediate and delayed tooth replantation and parameters receiving scores $1-4,1$ represent best outcomes, $2-3$ represent intermediate outcomes, and 4 represents the worst outcome (Holland et al., 1999, 2007). Data were submitted to statistical analysis using the Mann-Whitney $U$-test to analyze the differences in each parameters and the characteristics in each group. The significance level was set at $5 \%$.

\subsection{Immunohistochemical analysis}

To perform immunohistochemical reactions, sections were submitted to indirect immunoperoxidase staining using polyclonal primary antibodies against TRAP (1:100, Goat TRAP Antibody, sc-30833, Santa Cruz Biotechnology), and PCNA (1:100, Mouse PCNA Antibody, VP-P980, Vector Laboratories, Burlingame, CA). Deparaffinized slides were incubated in a citrate buffer solution $(\mathrm{pH}$ 6.0) in an equipment pressurized incubator (Decloaking Chamber, Biocare Medical, Concord, CA) with the temperature adjusted to $95^{\circ} \mathrm{C}$ for $20 \mathrm{~min}$. Subsequently, the slides were treated with hydrogen peroxide (Merck KGaA, Darmstadt, Germany) for $1 \mathrm{~h}$ and albumin bovine serum (Sigma-Aldrich) at 3\% for 1 additional hour. The histologic sections were incubated for $24 \mathrm{~h}$ in phosphate-buffered saline solution. Later, octoxynol-9 (Triton X100 , Sigma-Aldrich) plus one of the primary antibodies were added. The biotinylated rabbit anti-goat (Dako, Carpinteria, CA)
$28 \mathrm{~d}$
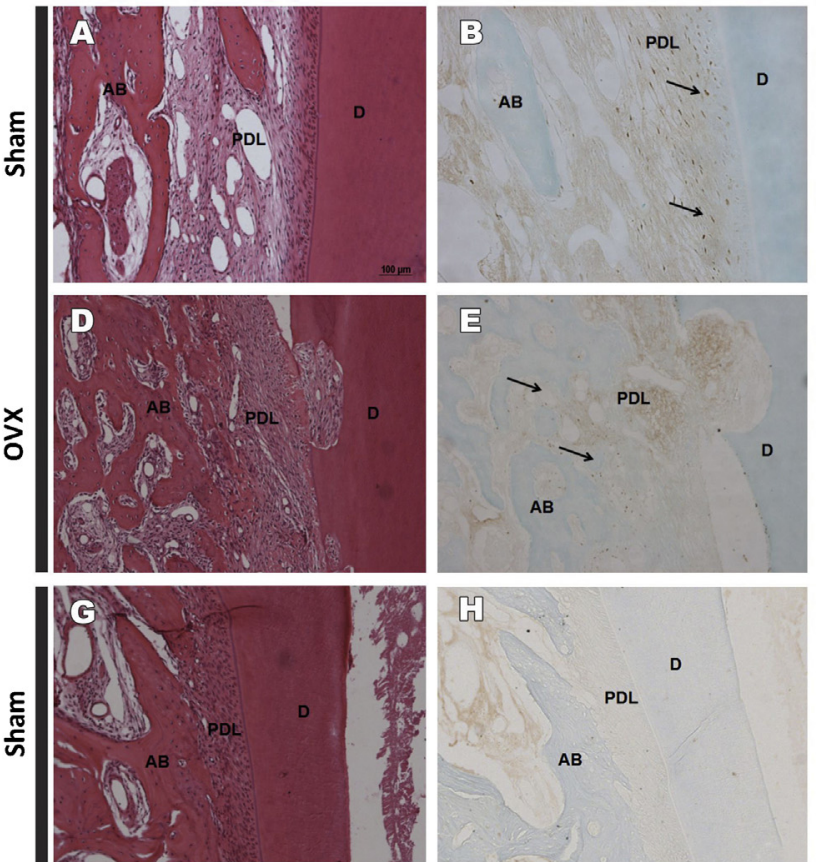

$45 d$
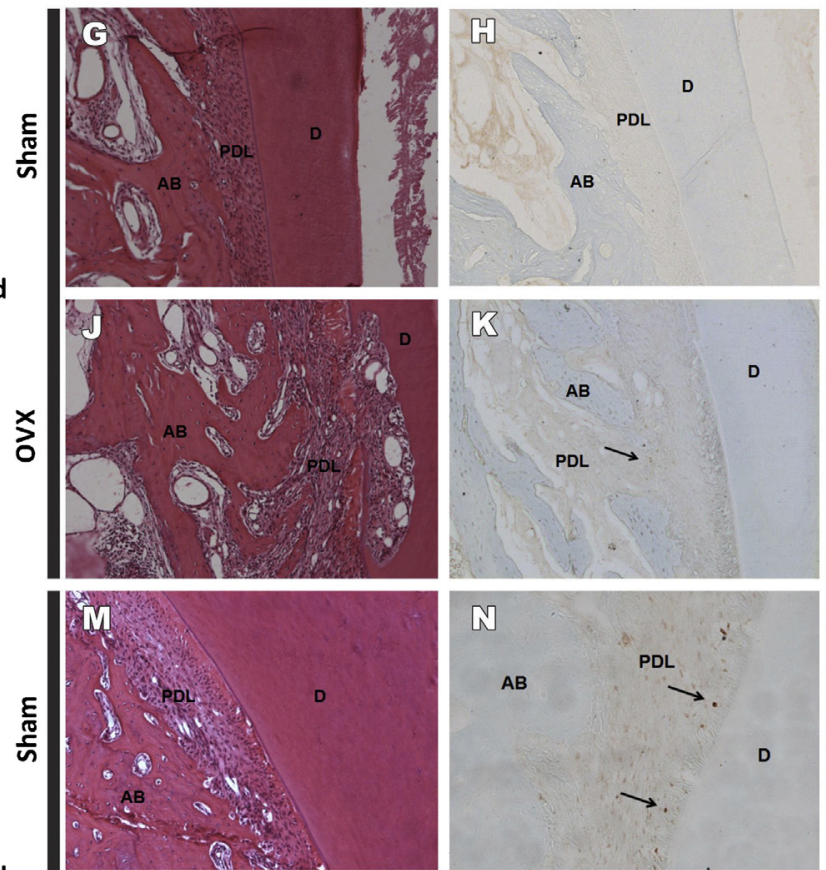

$60 \mathrm{~d}$

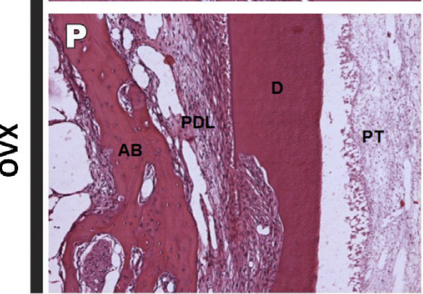

(ब)

$A B$

PDL

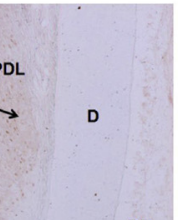

PT

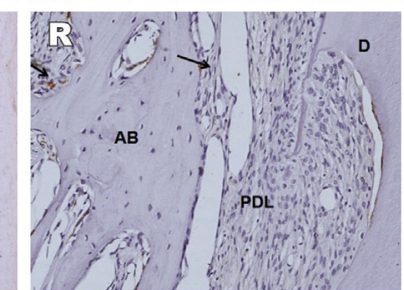

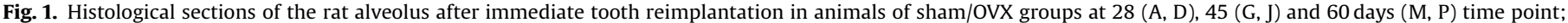

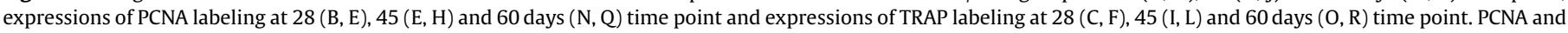
TRAP = arrow. Dentin (D), alveolar bone (AB), periodontal ligament (PDL), pulp tissue (PT). Scale bar (10 $\mu \mathrm{m})$. 
was used as a secondary antibody for 1 additional hour. Streptavidin-biotin-peroxidase complex (StreptAB-Complex/HRP, Dako) was then used for $1 \mathrm{~h}$ to amplify the signal of the reaction developed with diaminobenzidine (Dako) as the chromogen. The procedure was completed by nuclear counterstaining using hematoxylin (TRAP) and fast green (PCNA). All samples were accompanied by a negative control. Immunostaining was evaluated along the alveolar bone, cementum, PDL and pulp with a digital camera (Axio Cam MRc5; Carl Zeiss do Brasil Ltda., Rio de Janeiro, RJ, Brazil) coupled to a stereomicroscope (Stemi 2000-C; Carl Zeiss do Brasil Ltda.).

The immunohistochemical markers of PCNA and TRAP were assessed by counting the TRAP-positive and PCNA-positive cells located at the cervical, middle and apical thirds in the cementum, PDL and alveolar bone. Only two areas of the dental pulp (middle and apical thirds) were selected to quantify the PCNA cells and the immunohistochemical markers of TRAP were not considered for the pulp since there are no osteoclatics cells in the pulp.

A masked trained examiner selected the sections for the histologic, histometric and immunohistochemical analyses. Another masked calibrated examiner conducted the data analysis. The values for each specimen section were measured three times by the same examiner on different days to reduce variability.

The number of PCNA positive immunoreactive cells in the pulp tissue was expressed as absolute average values/1 $\times 105 \mu \mathrm{m}$ (Andreasen et al., 1995) in relation to the pulp area selected for the quantification. The same method was used to evaluate the number of PCNA immunoreactive cells in the periodontal ligament.
The number of PCNA positive immunoreactive cells was expressed as absolute average values $/ 1 \times 102 \mu \mathrm{m}$ in relation to the perimeter of the cementum (dental root surfaces) and alveolar bone. The same analysis was performed to quantify the TRAP positive cells in the cementum and in the alveolar bone. The number of TRAP positive immunoreactive cells in the periodontal ligament was expressed as absolute average values/ $1 \times 105 \mu \mathrm{m}$ (Andreasen et al., 1995 ) in relation to the area of PDL selected for the quantification.

The intensity of labeled cells in each glass slide was assessed using the National Institutes of Health image analyzer software (Image 1.410 , National Institutes of Health, USA). Data were submitted to statistic analysis using analysis of variance (ANOVA). The significance level was set up at $5 \%$.

\section{Results}

\subsection{Histological and immunohistochemical analysis}

\subsubsection{Sham/OVX group 28 days}

The sub-epithelial connective tissue showed few inflammatory infiltrate and epithelial attachment was present at the cementoenamel junction. The PDL fibers were reinserted into the cementum and bone tissue and it was intact in most of the root surface (Fig. 1A). In the OVX group, resorption areas of cementum and dentin were noticed (Fig. 1D). The pulp tissue was amorphous and the odontoblast layer along the middle and cervical thirds were irregular for both groups. In the apical area, intense acute
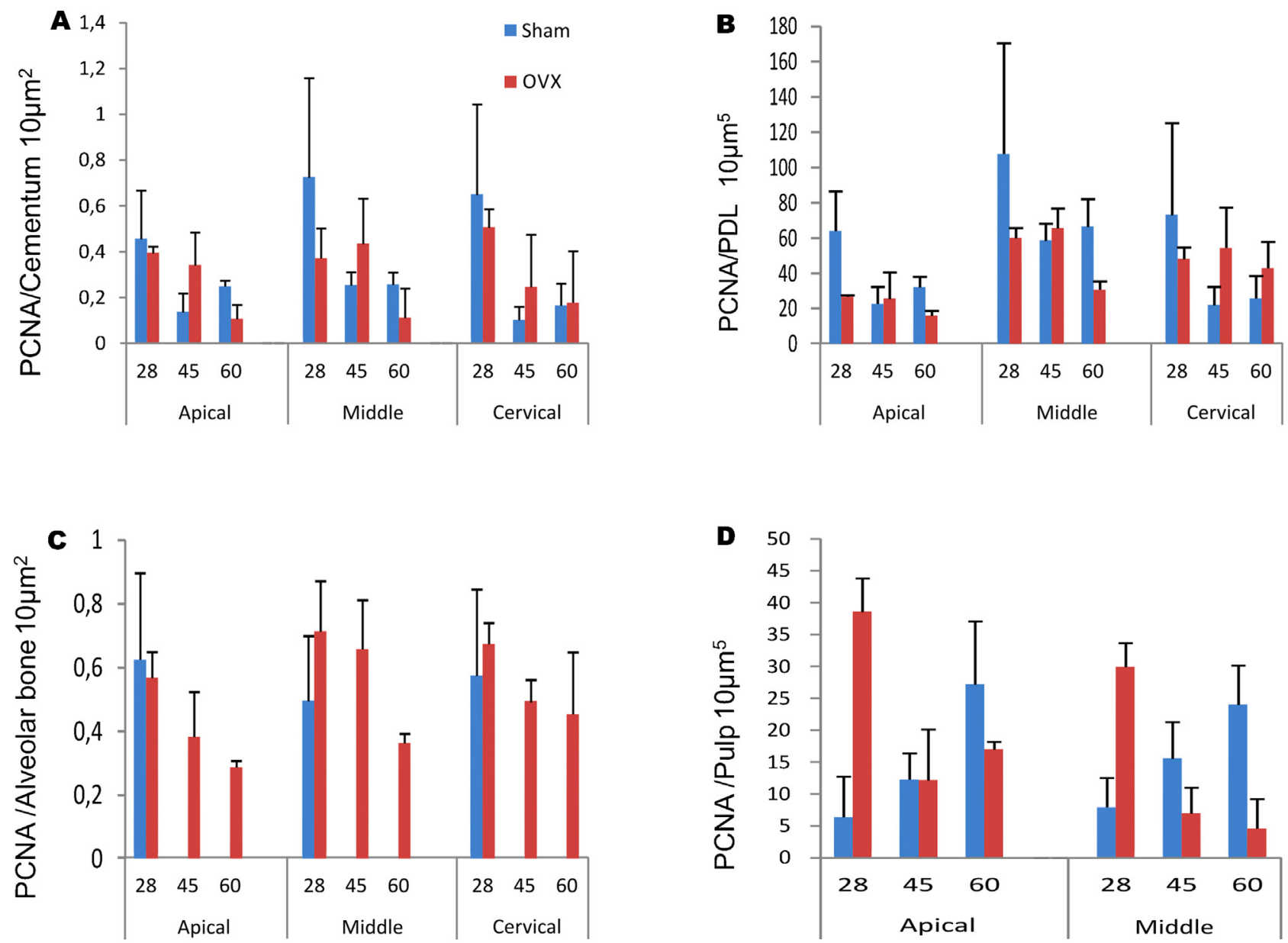

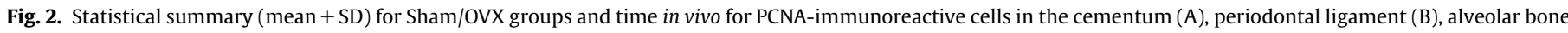
(C) and pulp (D) 
inflammatory infiltrate was observed, which invaded the root canal.

Immunoreactive cells for PCNA in both groups revealed intense positive immunostaining in the periodontal ligament (Fig. 1B). There were positive cells for PCNA in the bone tissue (Fig. 1E), while in the cementum and pulp tissue the immunostaining was weaker. Immunoreactive cells for TRAP were in the PDL and alveolar bone are also presented (Fig. 1C and F).

\subsubsection{Sham/OVX group 45 days}

Epithelial attachment was observed at the cemento-enamel junction for all the groups. The sub-epithelial connective tissue showed inflammatory infiltrate along the entire root and the PDL healed in most of the specimens (Fig. 1G). Root resorption was present in the OVX group (Fig. 1J) and the pulp tissue appeared degenerated without inflammatory cells.

Immunoreactive cells for PCNA, revealed reduced positive immunostaining in the periodontal ligament for both groups (Fig. $1 \mathrm{H}$ and $\mathrm{K}$ ). The positive cells were more evident in the PDL for OVX group (Fig. $1 \mathrm{~K}$ ) and were decreased in the pulp and cementum tissue for both groups. Immunoreactive cells for TRAP were present in the alveolar bone (Fig. 1I and L) for both groups and in the resorption areas of root surface for OVX group (Fig. 1L).

\subsubsection{Sham/OVX group 60 days}

The sub epithelial connective tissue showed few inflammatory infiltrate. The PDL space was diminished and filled by new bone formation in the Sham group (Fig. 1M). The OVX group showed resorption areas of the dentin and cementum (Fig. 1P). The pulp tissue was degenerated and the inflammatory infiltrate cells invaded the root canal in the apical area.

There were a decreased number of positive PCNA-cells in this period. Some positive cells were notice in the PDL for both groups (Fig. 1N and Fig. 1Q) and a decreased of positive cells in the cementum and pulp tissue was observed. The immunoreactive cells for TRAP were presented in the PDL and alveolar bone (Fig. 10 and Fig. 1R) for both groups.

\subsubsection{Histomorphometric and immunohistochemical analysis}

A significant difference in the PDL and root resorption histomorphometric parameters was observed. For the sub-item PDL organization, the significant difference were presented at 28 days $(p<0.04)$ and for the sub-item intensity of chronic inflammation, the significant difference was noticed at 60 days $(p<0.005)$ in vivo. For the sub-item depth of root resorption, the significant difference was observed at 45 days $(p<0.017)$ and at 60 days $(p<0.0006)$ in vivo. PCNA and TRAP proteins showed no significant differences between groups and times in vivo (Figs. 2 and 3).

\section{2. $\mu C T$ analysis}

\subsubsection{Sham/OVX groups 28 days}

Root resorptions and bone resorptions were presented in the apical and middle regions of the root respectively (Fig. 4A and B).

\subsubsection{Sham/OVX groups 60 days}

Most specimens presented root resorption and bone resorption in the apical and middle regions of the root (Fig. 4C and D). However, in the OVX group, the bone resorptions were noticed in the entire root surface, whereas in the Sham group was presented in the middle region of the root.

Fig. 4 depicts the statistical summary of root resorption extension, periodontal ligament space, bone resorption and depth of root resorption for both groups at 28 and 60 days. At 60 days, Sham and OVX groups presented higher root resorption extension relative to 28 days with higher values for OVX group (Fig. 4E). However, higher values for Sham group (Fig. 4F) was observed relative to OVX group at both time in vivo. At the 60 days, the Sham and OVX groups presented higher values for bone resorption relative to 28 days with higher values for OVX group (Fig. 4G). An increase in depth of root resorption mean values was observed for OVX groups at both times in vivo. At 60 days, Sham and OVX group presented higher values relative to 28 days (Fig. $4 \mathrm{H}$ ). No significant differences between groups were detected at both times in vivo for root resorption extension, periodontal ligament space, bone resorption and depth of root resorption.

\section{Discussion}

Research on humans and laboratory animal subjects have shown contradictory results in terms of the influence of estrogen deficiency on alveolar bone loss (Dervis, 2005; Aydim et al., 2013). One reason is that the effect of ovariectomy on bone tissue is not immediate and generally takes more than three months to be observed (Kuroda et al., 2003). However, Yoon et al. (2012) reported increased bone turnover and significant trabecular bone loss in ovariectomized rats after 8 weeks in the spine.

This study showed the ovariectomy did not have significant influence on immediate tooth reimplantation in rats. One possible reason is that the occlusal force, due to rat behavior, may have influenced the expected ovariectomy effect on the mandible (Mavropoulos, Rizzoli, \& Ammann, 2007; Ejiri, Toyooka, Tanaka, Anwar, \& Kohno, 2006). The mechanical load on the alveolar
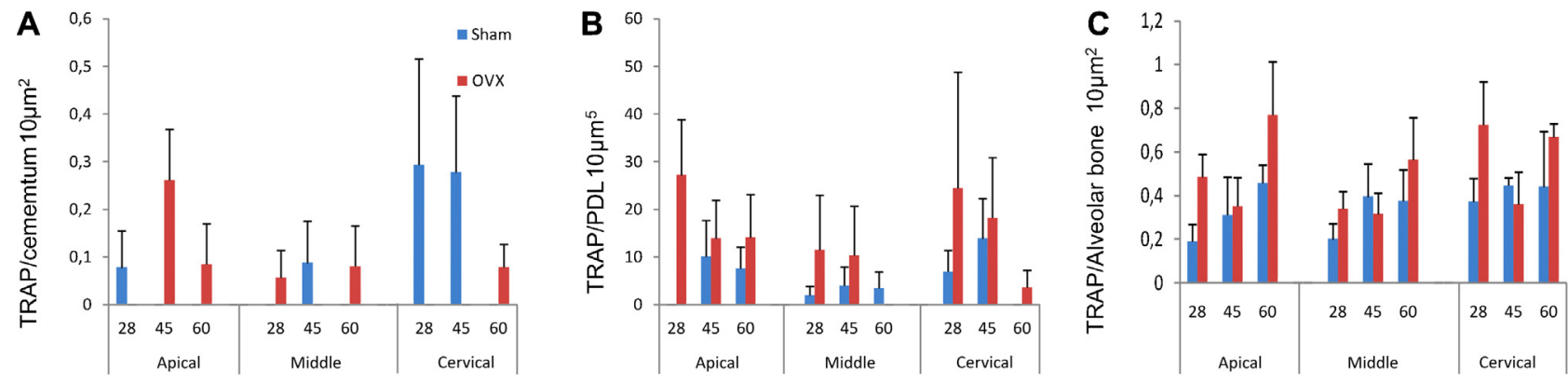

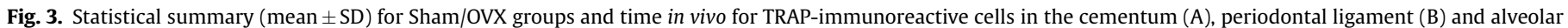
bone (C). 

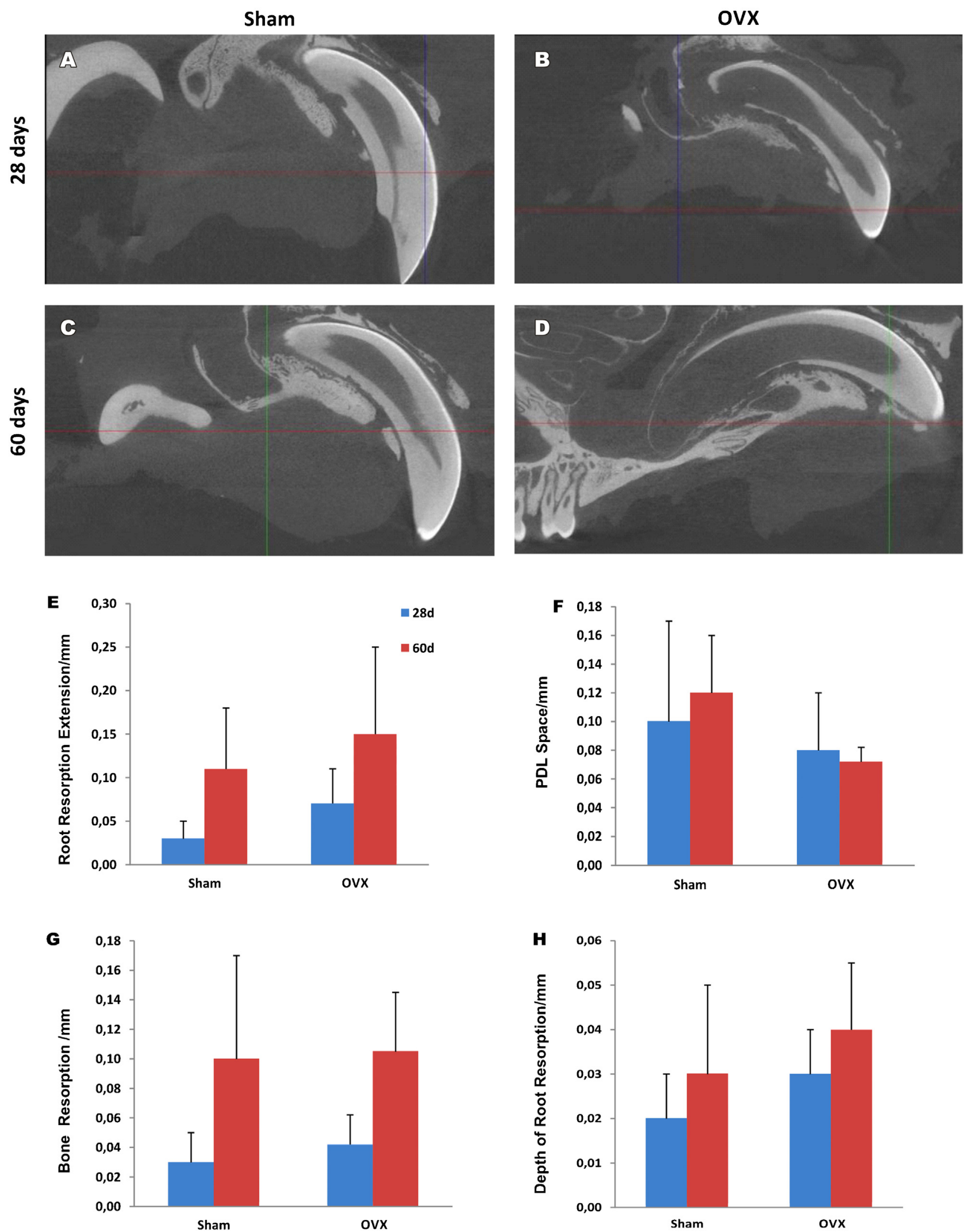

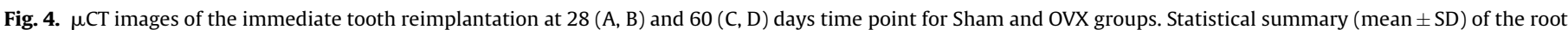
resorption extension (E), periodontal ligament space $(F)$, bone resorption $(G)$ and depth of root resorption (H) for Sham/OVX groups at both times in vivo.

process during chewing can protect the alveolar bone from the negative effects, which is not observed in other parts of the skeleton, such as the tibia (Mavropoulos et al., 2007) and the spine (Yoon et al., 2012). 
Morphological and embryonic variations between the skeletal regions may have also have had influences. Mavropoulos et al. (2007) reported an increase in tibia bone loss when compared to the alveolar bone in ovarectomized rats (Mavropoulos et al., 2007). Kim et al. (2012) claimed the trabecular bone mineral density variability, resulting from bone remodeling in the ovariectomzed rats, influences the vertebrae deformation (Kim et al., 2012).

The periodontium consists of the periodontal ligament, cementum and alveolar bone and play an important role in maintaining the mechanical stability of the teeth. Masticatory forces applied to teeth are transferred directly to the alveolar bone through the PDL, which results in bone remodeling. This bone remodeling changes the properties of the bone tissue when compared to other areas of the mandible that did not receive this force (Ames et al., 2010).

Regarding to the tooth reimplantation healing, all the groups presented periodontal ligament reinserted into the bone and cementum and few areas of root resorption at 28 days, whereas the root resorption areas were evident after 45 days. The PDL reinsertion was due to the viability of their cells at the time of reimplantation, which allowed for root regeneration (Andreasen, 1980; Andreassen, 1981; Hammarström, Blomlöf, \& Lindskog, 1989; Pohl, Filippi, \& Kirschner, 2005).

PCNA staining was performed to elucidate the effect of the estrogen deficiency on the osteoblasts and fibroblasts compared to the Sham group. PCNA is a cell cycle-related nuclear protein that is maximally elevated in the late $\mathrm{G} 1$ and $\mathrm{S}$ phases of proliferating cells. In our study, the rate of PCNA-positive cells in the in cementum, PDL, alveolar bone and pulp was more evident at 28 days for both groups.

The increase in the TRAP-positive cells for the OVX group at 28, 45 and 60 days post-treatment, mainly in the bone and periodontal ligament is consistent with previous studies that showed augmentation of osteoclast induced following estrogen deficiency in vivo (Luvizuto et al., 2010). Reduced levels of TRAP staining was observed in the cementum showing that there is cementoblast preservation which do not have receptors for the clastic cells and are essential for root preservation (Andreasen, 1980; Andreassen, 1981; Hammarström et al., 1989; Pohl et al., 2005; Consolaro, 2013).

In immediate tooth reimplantation with incomplete root formation, pulp revascularization may occur, thus it is not recommended the endodontic treatment immediately after tooth reimplantation (Andreasen and Andreasen, 2007; Andersson et al., 2012). In this study, all specimens at 28, 45 and 60 days showed degeneration of pulp tissue without contamination. This explains the absence of acute inflammatory infiltration and therefore the inflammatory root resorption. In clinical practice, teeth presenting this condition should be periodically monitored due to the risk of the pulp contamination and pulp degeneration that can lead to the development of inflammatory root resorption and the tooth loss.

Histomorphometric and immunohistochemical evaluation showed the root resorption areas were concentrated at the apical third while bone resorption areas were concentrated at middle and apical thirds for the both groups. In general, these are the areas most affected by resorption as a result of the inflammatory process generated by the trauma avulsion and replantation (Andreasen et al., 1995).

The PDL space was greater in the Sham group, which can be explained by the maintenance of the periodontal ligament between the alveolar bone and the cementum in the most of the specimens (Ho et al., 2009). However, the PDL space was smaller in the OVX group due to narrowing of the periodontal ligament, likely due to bone repair processes.

Even with a viable periodontal ligament, some areas can undergo necrosis, leading to the loss of cells such as pre-cementoblasts and cementum. The replacement of cementoblasts by osteoblasts during the healing process would result in a higher concentration of local or systemic inflammatory mediators. Inflammatory mediators manage the unit cells osteo-remodeling on the root surface, causing the teeth to undergo bone remodeling (Consolaro, 2013).

It is known that the estrogen deficiency down regulates protein expression while causing the release of cytokines such as IL-6 and TNF- $\alpha$ that play a destructive role in bone tissue (Zhou et al., 2009). Since such condition was simulated in out animal model, the estrogen deficiency may possibly explain the differences between the Sham and OVX groups in relation to inflammation processes, depth of root resorption, and osteoclastogenesis. However, such mechanisms should be further elucidated in future studies.

\section{Conclusion}

The results presented herein suggest that the decrease in estrogen did not contribute to a significant change in the multiple periodontal tissue phenotypes following tooth replantation.

\section{Acknowledgements}

We thank FAPESP for the financial support that enabled the authors to develop this study (process \#: 2010/12593-8, 2011/09295-8 and 2012/08254-9), Center for Craniofacial Regeneration, Columbia University College of Dental Medicine, and the Department of Biomaterials \& Biomimetics, New York University.

\section{References}

Ames, M. S., Hong, S., Lee, H. R., Fields, H. W., Johnston, W. M., \& Kim, D. G. (2010). Estrogen deficiency increases variability of tissue mineral density of alveolar bone surrounding teeth. Archives of Oral Biology, 55(8), 599-605.

Andersson, L., Andreasen, J. O., Day, P., Heithersay, G., Trope, M., Diangelis, A. J., et al. (2012). International Association of Dental Traumatology guidelines for the management of traumatic dental injuries: 2. Avulsion of permanent teeth. Dental Traumatology, 28(2), 88-96.

Andreasen, F. M., \& Andreasen, J. O. (2007). Avulsions, In J. O. Andreasen, F. M Andreasen, \& L. Andersson (Eds.), Textbook and color atlas of traumatic injuries to the teeth (pp. 444-488).4th ed. Oxford: Blackwell Munksgaard.

Andreasen, J. O., Borun, M. K., Jacobsen, H. L., \& Adreasen, F. M. (1995). Replantation of 400 avulsed permanent incisors: 2. Factors related to pulpal healing. Endodontics and Dental Traumatology, 11(2), 59-68.

Andreasen, J. O. (1980). A time-related study of periodontal healing and root resorption activity after replantation of mature permanent incisors in monkeys. Swedish Dental Journal, 4(3), 101-110.

Andreassen, J. O. (1981). Effect of extra-alveolar period and storage media upon periodontal and pulpal healing after replantation of mature permanent incisors in monkeys. International Journal of Oral Surgery, 10(1), 43-53.

Aydim, A., Kenar, H., Atmaca, H., Alici, T., Gacar, G., Muezinoglu, U. S., et al. (2013). The short- and long- term effects of estrogen deficiency on apoptosis in musculoskeletal tissues: an experimental animal model study. Archives of Iranian Medicine, 16(5), 271-276.

Consolaro, A. (2013). The four mechanisms of dental resorption initiation. Dental Press Journal of Orthodontics, 18(3), 7-9.

Dervis, E. (2005). Oral implications of osteoporosis. Oral Surgery, Oral Medicine, Oral Pathology, Oral Radiology and Endodontics, 100(3), 349-356.

Ejiri, S., Toyooka, E., Tanaka, M., Anwar, R. B., \& Kohno, S. (2006). Histological and histomorphometrical changes in rat alveolar bone following antagonistic tooth extraction and/or ovariectomy. Archives of Oral Biology, 51(11), 941-950.

Girasole, G., Passeri, G., Pedrazzoni, M., Giuliani, N., \& Passeri, M. (1995). Interleukin6: apathogenetic role in the post-menopausal osteoporosis? Acta Bio-Medica de 1 Ateneo Parmense, 66(3-4), 125-138.

Hammarström, L., Blomlöf, L., \& Lindskog, S. (1989). Dynamics of dentoalveolar ankylosis and associated root resorption. Endodontics and Dental Traumatology, 5(4), 163-175

Ho, S. P., Yu, B., Yun, W., Marshall, G. W., Ryder, M. I., \& Marshall, S. J. (2009). Structure, chemical composition and mechanical properties of human and rat cementum and its interface with root dentin. Acta Biomaterialia, 5(2), 707-718.

Holland, R., Souza, V., Nery, M. J., Otoboni Filho, J. A., Bernabé, P. F., \& Dezan Júnior, E. (1999). Reaction of rat connective tissue to implanted dentin tubes filled with mineral trioxide aggregate or calcium hydroxide. Journal of Endodontics, 25(3), $161-166$. 
Holland, R., Mazuqueli, L., de Souza, V., Murrata, S. S., Dezan Júnior, E., \& Suzuki, P. (2007). Influence of the type of vehicle and limit of obturation on apical and periapical tissue response in dogs teeth after root canal filling with mineral trioxide aggregate. Journal of Endodontics, 33(6), 693-697.

Jabbar, S., Drury, J., Fordham, J. N., Datta, H. K., Francis, R. M., \& Tuck, S. P. (2011). Osteoprotegerin, RANKL and bone turnover in postmenopausal osteoporosis. Journal of Clinical Pathology, 64(4), 354-357.

Kim, D. G., Navalgund, A. R., Tee, B. C., Noble, G. J., Hart, R. T., \& Lee, H. R. (2012) Increased variability of bone tissue mineral density resulting from estrogen deficiency influences creep behavior in rat vertebral body. Bone, 51(5), 868-875.

Kuroda, S., Mukohyama, H., Hondo, H., Aoki, K., Ohya, K., Ohyama, T., et al. (2003). Bone mineral density of the mandible in ovariectomized rats: analyses using dual x-ray absorptiometry and peripheral quantitative computed tomography. Oral Diseases, 9(1), 24-28.

Kwak, H. B., Lee, B. K., Oh, J., Yeon, Jt., Choi, S. W., Cho, H. J., et al. (2010). Inhibition of osteoclast differentiation and bone resorption by rotenone, through downregulation of RANKL-induced c-Fos and NFATc1 expression. Bone, 46(3), 724731.

Liang, L., Yu, J. F., Wang, Y., \& Ding, Y. (2008). Estrogen regulates expression of osteoprotegerin and RANKL in human periodontal ligament cells through estrogen receptor beta. Journal of Periodontology, 79(9), 1745-1751.

Luvizuto, E. R., Dias, S. M., Queiroz, T. P., Okamoto, T., Garcia, I. R. Jr., Okamoto, R., et al. (2010). Osteocalcin immunolabeling during the alveolar healing process in ovariectomized rats treated with estrogen or raloxifene. Bone, 46(4), 1021-1029.

Marão, H. F., Panzarini, S. R., Aranega, A. M., Sonoda, C. K., Poi, W. R., Esteves, J. C., et al. (2012). Periapical tissue reactions to calcium hydroxide and MTA after external root resorption as a sequela of delayed tooth replantation. Dental Traumatology, 28(4), 306-313.

Mavropoulos, A., Rizzoli, R., \& Ammann, P. (2007). Different responsiveness of alveolar and tibial bone to bone loss stimuli. Journal of Bone and Mineral Research, 22(3), 403-410.
Panzarini, S. R., Holland, R., de Souza, V., Poi, W. R., Sonoda, C. K., \& Pedrini, D. (2007). Mineral trioxide aggregate as a root canal filling material in reimplanted teeth: microscopic analysis in monkeys. Dental Traumatology, 23(5), 265-272.

Panzarini, S. R. Okamoto, R. Poi, W. R., Sonoda, C. K., Pedrini, D., da Silva, P. E., et at. (2013). Histological and immunohistochemical analyses of the chronology of healing process after immediate tooth replantation in incisor rat teeth. Dental Traumatology, 29(1), 15-22.

Pfeilschifter, J., Koditz, R., Pfohl, M., \& Schatz, H. (2002). Changes in proinflammatory cytokine activity after menopause. Endocrine Reviews, 23(1), 90-119.

Pohl, Y., Filippi, A., \& Kirschner, H. (2005). Results after replantation of avulsed permanent teeth: II. Periodontal healing and the role of physiologic storage and antiresorptive-regenerative therapy. Dental Traumatology, 21(2), 93-101.

Riggs, B. L., Khosla, S., \& Melton, L. J. III (2002). Sex steroids and the construction and conservation of the adult skeleton. Endocrine Reviews, 23(3), 279-302.

Said, F., Ghoul-Mazgar, S., Khemiss, F., El Ayebe, H., Saidane, D., \& Berdal, A. (2012), The effect of etidronate on the periodontium of ovariectomized rats. Journal of Periodontology, 83(8), 1063-1068.

Song, L., Zhang, X., \& Zhou, Y. (2011). A synergetic role of 1,25-dihydroxyvitamin D (3) in 17b-estradial inducedproliferation and differentiation of osteoblastic MC3T3-E1 cells. European Journal of Pharmacology, 659(2-3), 273-280.

Theoleyre, S., Wittrant, Y., Tat, S. K., Fortun, Y., Redini, F., \& Heymann, D. (2004). The molecular triad OPG/RANK/RANKL: involvement in the orchestration of pathophysiological bone remodeling. Cytokine and Growth Factor Reviews, 15(6), 457-475.

Yoon, K. H., Cho, D. C., Yu, S. H., Kim, K. T., Jeon, Y., \& Sung, J. K. (2012). The change of bone metabolism in ovariectomized rats: analyses of MicroCT scan and biochemical markers of bone turnover. Journal of Korean Neurosurgical Society, 51(6), 323-327.

Zhou, Y., Fu, Y., Li, J. P., \& Qi, L. Y. (2009). The role of estrogen in osteogenetic cytokine expression in human periodontal ligament cells. International Journal of Periodontics and Restorative Dentistry, 29(5), 507-513. 